\title{
Evaluation of the outcomes of right paralumbar fossa colostomy for type II colonic atresia in calves: a report of 24 cases
}

\author{
MUHARREM EROL, HANIFI EROL*, SEMIH ALTAN**, NURI YAVRU*** \\ Balıkesir University, Veterinary Faculty, Surgery Department, Balıkesir/Turkey \\ *Erciyes University, Veterinary Faculty, Surgery Department, Kayseri/Turkey \\ **Dicle University, Veterinary Faculty, Surgery Department, Diyarbakır/Turkey \\ ***Selcuk University, Veterinary Faculty, Surgery Department, Konya/Turkey
}

Erol M., Erol H., Altan S., Yavru N.

Evaluation of the outcomes of right fossa paralumbar colostomy for type II colonic atresia in calves: a report of 24 cases

Summary

The aim of the present study was to evaluate the effectiveness of right fossa paralumbar colostomy on the survival rate of calves with atresia coli maintained under Anatolian farming conditions. The clinical diagnosis was confirmed by clinical findings, pain and contrast radiographic examination. In clinical and indirect radiographic examination, rectum and descending colon were seen as more constricted than usual. In surgery, the abdominal cavity was opened through a vertical incision from the right paralumbar fossa. The blind end of the ascending colon was fixed to the abdominal wall in order to create a stoma. The postoperative course of the calves was obtained from the owners of the calves during a period of four months. It was learned that only one calf survived and the others died because of diarrhea within the four month period. In conclusion, this study demonstrated that although right fossa paralumbar colostomy was successfully performed for type-II atresia coli, severe functional impairment and fluid imbalance due to the loss of an important fluid resorption resulted in the death of most calves that were kept under Anatolian farming conditions.

Keywords: atresia, calves, colostomy

Atresia is a condition in which an orifice or passage in the body is abnormally closed or absent. The known types of atresia involving the intestines are named after their location. This condition has been adequately described in cattle $(9,11,12)$. While atresia ani is believed to be hereditary, atresia coli is a consequence of congenital adverse events and is characterized by complete occlusion of the intestinal lumen due to anomalous development of the intestinal wall. Atresia coli is believed to be a secondary effect of the disruption of fetal intestinal blood supply in the organogenesis period $(15,16)$. Some authors define the rigorous rectal palpation as a potential risk factor for atresia coli in calves. If not treated, atresia coli can be lethal. Generally, affected calves appear normal at birth. However, clinical symptoms develop soon after they begin drinking milk, with abdominal distension being one of the first symptoms. Furthermore, no feces are passed. Animals become anorexic and increasingly weak and lethargic and eventually show signs of colic a few days after birth $(3,6)$. Radiographic imaging shows gas-filled intestinal structures and retrograde contrast radiography shows blind ended sacs (15). All untreated animals die, except those with passable rectovaginal fistulae (7). Moreover, chances of complete healing after the surgical treatment of atresia coli have been reported to be poor (9-11). On the other hand, Azizi et al. (4) reported a $>6$ month survival rate of $73 \%$ for calves with atresia coli treated by colostomy. In the Anatolian region new born calves often die due to atresia coli.

The aim of the present study was to evaluate the effect of right paralumbar fossa colostomy on the survival rate of calves with atresia coli maintained under Anatolian farming conditions.

\section{Material and methods}

In total, 23 Holstein Friesian calves and one Brown Swiss calf with atresia coli were referred to the Large Animal Clinic at the Faculty of Veterinary Medicine of Selcuk 
University. The mean age of calves $[ \pm$ standard deviation (SD)] was $3.7 \pm 0.4$ days. There were 20 male and four female calves (Tab. 1). The diagnosis was based on the absence of feces, findings of digital palpation, progressive weakness, and abdominal distension. The presence of thick, yellowish or white mucus in the rectum indicates atresia. The diagnoses for all calves were confirmed through direct abdominal radiography and contrast radiography. Radiographic findings included distension of the small and large intestines by gas, which was present in all animals (Fig. 1A). The blind ends of colon or rectum were visualized radiographically after the administration of barium enemas. Retrograde radiography with barium contrast also showed the blind part of the colon (Fig. 1B, C, D). Venous blood gas analysis was performed and the white blood cell count was measured before and after surgery. The results are shown in Table 2.

Intravenous (IV) fluids (Ringer lactate, 3 ml/kg b.w., Polifarma, Ankara, Turkey) were administered to correct acid-base and electrolyte imbalances and remedy dehydration. Intramuscular (IM) procaine penicillin $(8 \mathrm{mg} / \mathrm{kg}$ b.w., Penokain-G, Vetaş, Istanbul, Turkey), dihydrostreptomycin $(10 \mathrm{mg} / \mathrm{kg}$ b.w., IM, Reptopen ${ }^{\circledR}$, Ceva-Dif, Istanbul, Turkey) and intravenous (IV) flunixin meglumine $(2.2 \mathrm{mg} / \mathrm{kg}$ b.w., IM, Fulimed,

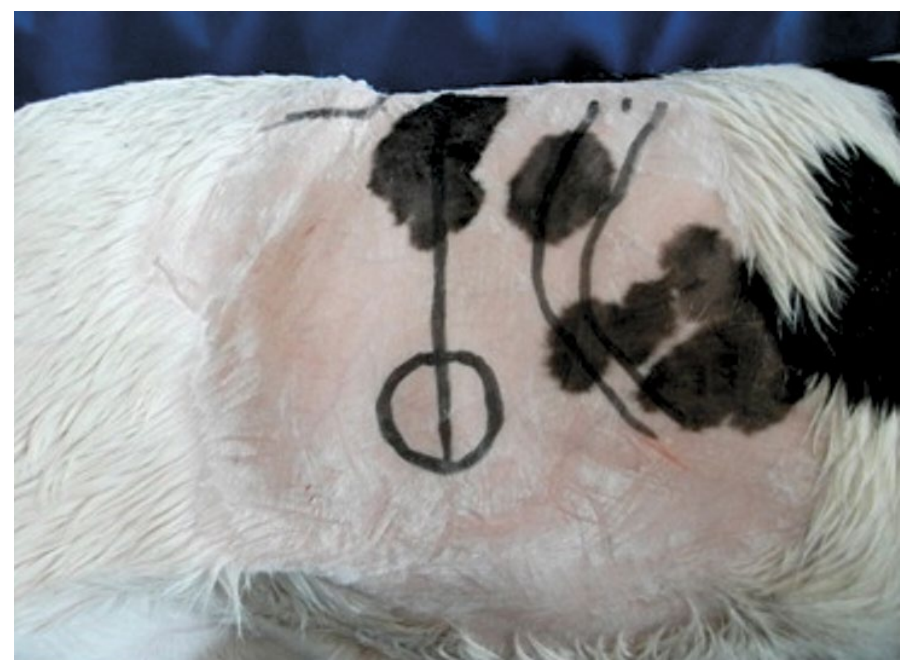

Fig. 2. Preparation of operation area for colostomy
Tab. 1. Signalement, surgical and clinical outcomes

\begin{tabular}{|c|c|c|l|l|}
\hline CN & BR & G & \multicolumn{1}{|c|}{ SO } & \multicolumn{1}{c|}{ CO } \\
\hline 24 & $23 \mathrm{HF}$ & $20 \mathrm{M}$ & $\begin{array}{l}\text { 20 discharged } \\
3 \text { owners not accept operation } \\
1 \text { deid during operation }\end{array}$ & $\begin{array}{l}10 \text { calves died between 1-20 days } \\
\text { 4 calves died one month later } \\
1 \text { calf died 3 months later } \\
1 \text { calf died 4 months later } \\
\text { 2 calves slaughtered 4 months later }\end{array}$ \\
\hline
\end{tabular}

Explanations: CN - number of calves; BR - breed; HF - Holstein-Friesian; BS - Brown-Swiss; G - gender; M - male; F - female; SO - surgical outcome; CO clinical outcome

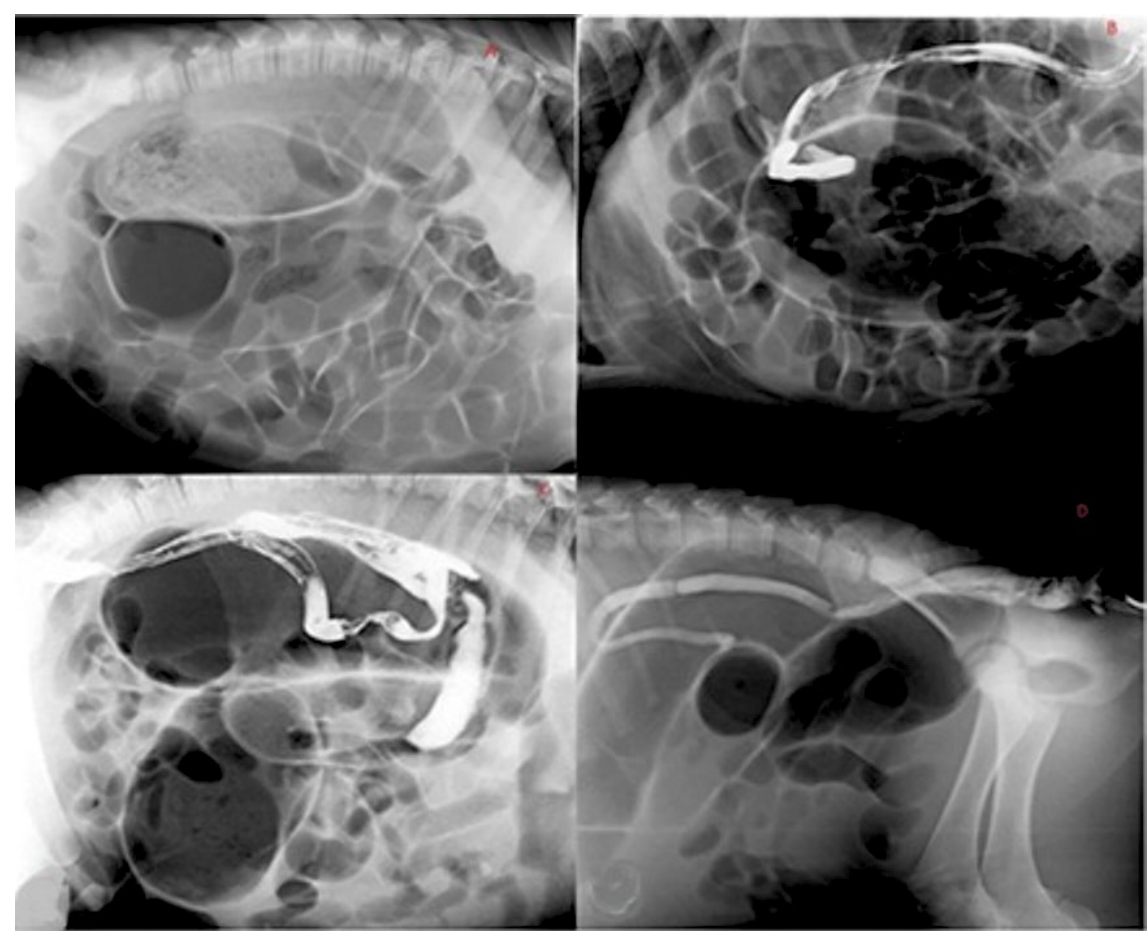

Fig. 1. Radiography of abdomen (A - direct radiography, $\mathrm{B}, \mathrm{C}, \mathrm{D}$ - retrograde contrast radiograpy)

Alke, Istanbul, Turkey) were administered prophylactically before surgery.

The sedation was achieved with $0.005 \mathrm{mg} / \mathrm{kg} \mathrm{b.w.,} \mathrm{IV}$ xylazine (Alfazyne, Ege-Vet, Izmir, Turkey), and $15 \mathrm{ml}, 2 \%$ lidocaine (Adokain, Sanovel, Istanbul, Turkey) was used for local infiltration anaesthesia. The animals were placed in left lateral recumbency, and the right paralumbar fossa was prepared for laparotomy (Fig. 2). The same pre-surgical procedure was performed for all animals.

Colostomy was performed according to the procedure described by Azizi et al. (4). A 10-12 cm skin incision was done in the right middle flank, which was followed by incision of the external abdominal oblique muscle. The internal abdominal oblique and transverse muscles were bluntly dissected in the direction of their fibres, and the perito-

Tab. 2. Venous blood gas analysis and hemocell count results (mean \pm SD)

\begin{tabular}{|c|c|c|c|c|c|c|c|c|c|}
\hline Day & $\mathrm{PH}$ & $\mathrm{PCO}_{2}$ & $\mathrm{PO}_{2}$ & $\mathrm{Na}$ & $\mathrm{K}$ & Lac & $\mathrm{Hct}$ & $\mathrm{HCO}_{3}^{-}$ & $\mathrm{WBC}^{-}$ \\
\hline Pre & $7.38 \pm 0.8$ & $49 \pm 5$ & $22 \pm 8$ & $142 \pm 4.3$ & $4.3 \pm 0.8$ & $4.2 \pm 3.5$ & $32 \pm 6.6$ & $29.5 \pm 6$ & $13.4 \pm 7.3$ \\
$\mathrm{P} 01$ & $7.37 \pm 0.7$ & $51 \pm 6$ & $19 \pm 5$ & $140 \pm 4.1$ & $4.2 \pm 0.6$ & $4.2 \pm 4.0$ & $30 \pm 5.0$ & $30.0 \pm 5$ & $14.7 \pm 5.5$ \\
\hline $\mathrm{P} 03$ & $7.36 \pm 0.7$ & $49 \pm 5$ & $22 \pm 7$ & $140 \pm 5.0$ & $4.3 \pm 0.8$ & $2.4 \pm 2.4$ & $32 \pm 6.0$ & $27.0 \pm 4$ & $14.6 \pm 8.0$ \\
\hline
\end{tabular}

Explanations: Pre - preoperative; $\mathrm{PO} 1$ - post-operative first day; $\mathrm{PO} 3$ - post-operative third day; $\mathrm{PH}$ - venous blood $\mathrm{PH}_{3} \mathrm{PCO}_{2}-$ partial venous blood carbondioxide; $\mathrm{PO}_{2}$ - partial venous oxygen pressure; $\mathrm{Na}$ - sodium; $\mathrm{K}$ - potassium; Lac - lactate; Hct - hematocrit; $\mathrm{HCO}_{3}$ - bicarbonate; WBC - white blood cell count 
neum was incised (Fig. 3A). After exploration of abdominal organs, the proximal blind end of the colon was exteriorized and fixed to the peritoneum and abdominal muscles distal to the mid flank incision. The colon was sutured in a circular fashion, using simple non penetrating interrupted absorbable suture material equidistant from both ends of the laparotomy incision (USB 2/0, Vicryl, Ethicon, AkınGlobal medical, Ankara, Turkey) (Fig. 3B). Next, the upper and lower parts of the abdominal wall incision were closed in layers. The blind end of the colon was incised, and after evacuation of the meconium and irrigation of the region with warm normal saline $(0.9 \% \mathrm{NaCl}$, Polifarma,

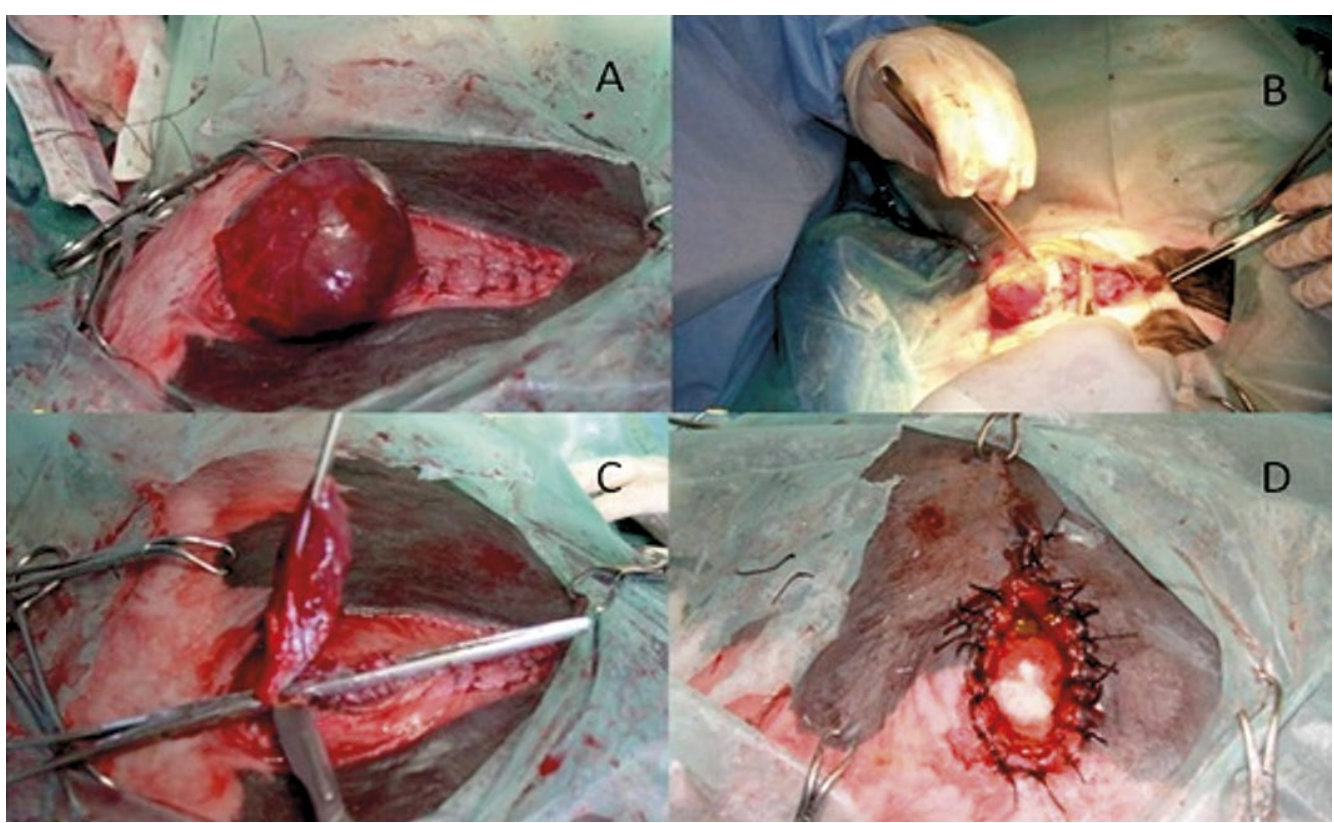

Fig. 3. Operation of right flank colostomy (A - blind end of colon after dissection, $B$ - suturing of colon to the abdominal muscle, $\mathrm{C}, \mathrm{D}$ - incision of blind part of colon and suturing to the skin)
Ankara, Turkey) solution, the intestinal wall was anchored to the skin using simple interrupted sutures (USB 0, Silk, Ethicon, AkinGlobal medical, Ankara, Turkey) (Fig. 3C, D). The skin sutures would be removed at 12-14 days after surgery by a farm veterinarian. After surgery, the calves were housed in a warm stall with dry bedding. For rehydration and correction of electrolyte imbalance, IV fluids were administered postoperative 2 days. The feeding was initiated $24 \mathrm{~h}$ after surgery, with small amounts of milk 2-3 times daily. Intramuscular same dosage penicillinstreptomycin was administered for 1 week after surgery, and flunixin meglumine was administered three times after surgery (every $24 \mathrm{~h}$ ).

The findings of venous blood gas analysis were analyzed using Shapiro-Wilk test for normality. One-way ANOVA followed by Tukey significant difference tests were used to compare preoperative and postoperative values. Statistical significance was accepted at $P<0.05$. All analyses were performed using SPSS 21 (IBM corp., USA) program. The results are presented as mean $\pm \mathrm{SD}$.

\section{Results and discussion}

All calves births were normal and they had consumed milk after birth. There were no pathological cases seen in their organs. However, the lack of defecation was a common complaint for the animals. In the clinical examination, abdominal distension, ping sound and meconium filled intestines were detected. After digital palpation and enema treatment, thick, yellowish or white mucus was obtained instead of feces. The hematologic values were within reference ranges, and no significant differences seen (Tab. 2). The increased amounts of peritoneal fluid and some fibrin deposits on the intestines were seen in the operations. The proximal blind end of the colon was found in the spiral colon loop.

One calf died 3 days after surgery. All other calves were discharged from the hospital after 3 days. The development of all animals was followed up by phone calls with the owners for a maximum of 4 months. Only one calf survived in that period, all the others died due to diarrhea (73\%). Eight calves died between 10 and 20 days after surgery due to poor physical condition and diarrhea, while nine calves survived 30 to 120 days (Tab. 1).

Congenital intestinal atresia is characterized by the complete closure of any segment of the intestinal tract $(5,6,15)$. Such anomalies have been reported in various animal species. Atresia coli is the most frequent malformation in dairy calves, and seen with a higher frequency in dairy calves than beef breeds $(5-7,15)$. Despite various attempts to establish a relationship between atresia coli and sex, there is no published data to support this relationship. In the present study, atresia coli were detected in 20 male (83.3\%) and $4(16.7 \%)$ female calves, this suggested that atresia coli is more common in male calves.

Atresia coli may be an inherited condition in HolsteinFriesian cattle. Their colon development rate is faster and/or of a greater extent than the other breeds during growth (4). It has been stated that amniotic palpation per rectum for the early diagnosis of pregnancy in cattle has the potential to disrupt organogenesis, particularly when performed before day 42 of gestation. Furthermore, it has consistently been associated with intestinal atresia $(4,8)$. In the anamnesis of our calves the pregnancy examinations had been performed at 40 days after artificial insemination was recorded. These findings support the increased risk of atresia coli due to pregnancy examination before 42 days in the Holstein Friesian breed.

The clinical signs of abdominal distension, decreased appetite, progressive depression, and failure to pass meconium are the indications of gastrointestinal obstruction, while detection of these findings in calves 
less than 8 days primarily suggest intestinal atresia (1, 13). With regard to the present study, we believe that the referral of 20 male and 4 female calves to our clinic was merely coincidental. The pathognomonic clinical sign, namely the presence of thick, yellowish or white mucus instead of meconium or feces in the rectum has been reported in previous studies. The clinical signs of four calves at the time of admission were typical, but additional diagnostic imaging was used to confirm the presumptive diagnosis of colonic atresia. Some studies emphasized that only contrast radiography was adequate to determine the site of atresia coli $(6,13,15)$. In the present study, retrograde contrast radiography showed a narrow and cord-shaped rectum lumen, as observed in other studies (14). ConsTab. et al. (6) did not identify a significant correlation between blood gas analysis and leukocyte counts in colonic atresia calves. Generally, the right flank laparotomy technique is used for differential diagnose of intestinal atresia. In the present study, there were no significant differences detected in blood analysis (Tab. 2), and colonic atresia was diagnosed after clinical examinations and retrograde contrast radiography, which were considered adequate for preoperative diagnosis. Finally, operative findings confirmed the diagnosis of colonic atresia.

Surgical treatment is not proposed in intestinal atresia calves due to economic considerations, low possibility of postoperative survival and the high risk of genetic transmission to the next generation $(6,11)$. However, many procedures are included for the creation of an anastomosis by using two viable ends, or colostomy technique, are used for the correction of colonic atresia in calves $(2,4)$. Either side-to-side or end-to-side anastomosis between the rectal or distal colon segments with the gut of the ansa proximalis of the ascending colon have been reported $(12,15)$. Steenhaut et al. (16) recommend right flank colostomy if the entire colon is absent.

In our study, type-II atresia calves were treated by the creation of a stoma to distally in the right flank. The surgical technique was thought to influence the survival rate (Tab. 1). Therefore, one calf died 3 days after surgery, eight died between 10 and 20 days after surgery due to poor physical condition, postoperative complications and diarrhea. Nine calves survived for 30 to 120 days. Smith et al. (15) used the anastomosis technique for the management of atresia in calves and $51 \%$ were successfully discharged. Thirty-seven percent of $51 \%$ reaching puberty was emphasized by Smith et al. (15). Koc et al. (12) used colostomy or the anastomosis technique and successfully discharged $54 \%$ of calves. In the present study, right flank colostomy technique was used for type-II atresia coli. The short-term survival rate was $75 \%$. The discharged calves mainly died $(78 \%)$ due to diarrhea. Although right flank colostomy is more successful than other techniques, the chances of success were low in the present study due to digestive tract infections. To prevent these complications after colostomy, suturing the proximal blind end of the colon as high as possible in the right flank is recommended. However, one disadvantage of this technique is extensive contamination of the flank which must be regularly cleaned (4). Collectively, the findings of previous studies and the present study demonstrate that even if colostomy techniques are successfully completed post-operative complications such as peritonitis, failure of anastomosis, diarrhea, incisional infection, chronic cecal dilatation, functional spiral colon obstruction, and adhesive bowel obstruction reduce the likelihood of long-term success. In the present study diarrhea was the most common cause of death. Thus, operative treatment of atresia coli is considered not economical $(3,12,15)$.

In conclusion, this study demonstrated that the right fossa paralumbar colostomy was successfully performed to type-II atresia coli. However, the severe functional impairment and fluid imbalance due to the loss of important fluid resorption resulted in the death of most calves that were kept under Anatolian farming conditions. Thus, the owners should be advised to refrain from surgical treatment for atresia coli. Even if the owners accept a surgical treatment technique such as right flank colostomy, important postoperative consequences should be clearly explained before undertaking the procedure.

\section{References}

1. Abouelelnasr K., Ishii M., Inokuma H., Kobayashi Y., Lee K., Yamada K. Atresia coli in a japanese black calf diagnosed by a barium sulphate enema contrast radiography in the standing position: a case report. Veterinarni Medicina 2012, 57, 376-379.

2. Alkan F., Koc Y., Ceylan C.: The surgical repair of calves with atresia coli. Indian Vet. J. 2002, 79, 841-843.

3. Atalan G., Ozaydın I., Kilic E., Cihan M., Kamiloglu A.: Buză̆ılarda intestinal atresia olguları ve operatif sağaltımları: 54 olgu (1992-2000). Kafkas Uni. Vet. Fak. Derg. 2003, 9, 113-118.

4. Azizi S., Mohammadi R., Mohammadpour I.: Surgical repear and management of congenital intestinal atresia in 68 calves. Vet. Surg. 2010, 39, 115-120.

5. Cecen G., Salci H., Caliskan G. U., Celimli N., Seyrek-Intas D., Görgül O. S.: Modified colostomy technique for colonic atresia in calves. Vet. Surg. 2010, 39, 722-728.

6. ConsTab. P. D., Rings D. M., Hull B. L., Hoffsis G. E., Robertson J. T.: Atresia coli in calves, 26 cases (1977-1987). J. Am. Vet. Med. Assoc. 1989, 195, 118 $-123$

7. Dreyfuss D. J., Tulleners E. P.: Intestinal atresia in calves, 22 cases (1978-1988). J. Am. Vet. Med. Assoc. 1989, 195, 508-513.

8. Göksel B. A., Sarıtaş Z. K.: Buzağılarda intestinal atresia'larda klinik ve operatif yaklaşımlar. Kocatepe Vet. J. 2016, 9, 200-210.

9. Hatch E. I., Schaller R. T.: Surgical management of multiple intestinal atresias Am. J. Surg. 1986, 151, 550-552.

10. Hoffsis G. F., Brunner R. R.: Atresia coli in a twin calf. J. Am. Vet. Med. Assoc. $1975,171,433-434$

11. Kilic N., Sarierler M.: Congenital intestinal atresia in calves: 61 cases (1999-2003). Reuve. Med. Vet. 2004, 155, 381-384.

12. Koc Y., Alkan F., Ceylan C., Birdane F. M.: Evaluation of clinical and surgical approaches in 22 calves with atresia coli (in Turkish). Vet. Bil. Derg. 2001, 17, 27-34.

13. Kumar R. V. S., Veena P., Sankar P., Laskhmi N. D., Kokila S.: Surgical management of atresiarecti-ani and coli in a holstein fresian cross bred calf. Veterinary World 2011, 4, 176-177.

14. Martens A., Gasthuys F., Steenhaut M., De Moor A.: Surgical aspect of intestinal atresia in 58 calves. Vet. Rec. 1995, 136, 141-144.

15. Smith D. F., Ducharme N. G., Fubini S. L., Donawick W. J., Erb H. N.: Clinical management and surgical repair of atresia coli in calves, 66 cases (1977-1988). J. Am. Vet. Med. Assoc. 1991, 199, 1185-1190.

16. Steenhaut M., De Moor A., Verschootnn F., Desmet P.: Intestinal malformations in calves and their surgical correction. Vet. Rec. 1976, 98, 131-133.

Corresponding author: Dr. Hanifi Erol, Erciyes University, Veterinary Faculty, Surgery Department, Kayseri, Turkey; e-mail: drhaneroll@yahoo. com 